\title{
Europe creates diagnostics centre
}

$\mathrm{U}$ nreliable, inaccurate or expensive diagnostic tests have long been identified as one of the biggest obstacles to containing infectious diseases in developing countries. Industry is viewed as reluctant to conduct research and development on needed new tests because the perceived return on investment is minimal. Many tests make it to the market without evidence of effectiveness because of a lack of regulation, and some say standards, within the field of diagnostics.

In a bid to redress those deficiencies, an International Diagnostics Centre (IDC) has been established at the London School of Hygiene \& Tropical Medicine in England to essentially twist the arms of nations to fast track the identification of biomarkers and the development of appropriate diagnostics for diseases that are prevalent in the developing world.

But the centre itself will not actually be researching or developing new diagnostic tests. Rather it will be seeking "to create a critical mass of researchers working across all different aspects of diagnostics from $R \& D$ [research and development] to costeffectiveness and delivery so that we can develop synergies and more effective collaborations," Rosanna Peeling, director of the IDC, and professor and chair of diagnostic research at the London School of Hygiene \& Tropical Medicine, writes in an email. "Members of the IDC can facilitate the development of new tests by identifying new diagnostic targets through molecular epidemiology or evaluating prototypes of new tests in collaboration with diagnostic companies."

The approach could be valuable, Dr. Lawrence Loh, adjunct lecturer in global health and epidemiology at the University of Toronto in Ontario, writes in an email. "Since global health research commonly occurs in silos, a successful centre such as this may well serve as a leading example of multidisciplinary, multi-centre collaboration that is so desperately needed."

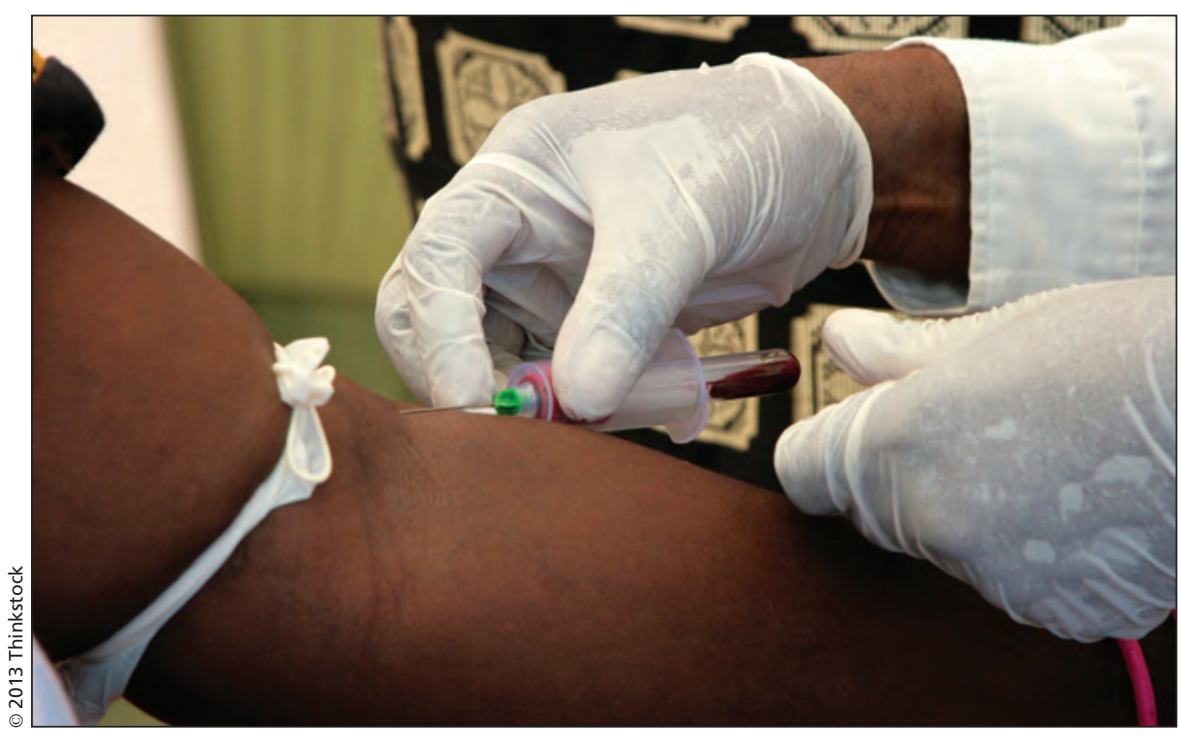

Ineffective diagnostic tests have been identified as a major obstacle to containing infectious diseases in developing countries.

Researchers from more than 100 countries will be asked to participate, many of them now involved in a current project to "improve the regulatory oversight of diagnostics in the developing world," Peeling adds.

Participating countries and sites will be expected to provide funding for initiatives aimed at rapid and inexpensive diagnostic tests for malaria, HIV and syphilis, as well as neglected tropical diseases such as trachoma, schistosomiasis, leishmaniasis and trypanosomiasis, Peeling writes.

"The current global diagnostics landscape is fragmented, lacks coordination and effective partnerships. An International Diagnostics Centre based at the school will bring together members of School staff and our UK [United Kingdom] and international partners working on different aspects of diagnostics, and provide them with a global platform for advocating the value of diagnostics, sharing information, promoting best practice and enhancing collaboration to develop and deliver innovative diagnostic tools to improve global health," she adds.

According to an IDC background presentation document, it takes between US\$10 million and US\$100 million, and between 2 to 12 years, to develop a diagnostic test, while "in 2004, investment in diagnostics represented less than $1 \%$ of the total global spending for malaria, compared to $37 \%$ for drug development and $24 \%$ for vaccines.

Loh believes the end result will be substantial improvements in patient care. "The benefits of point of care testing could therefore greatly affect the burden of these diseases targeted by the centre - by helping patients get the treatment they need faster, and by being able to propagate testing efforts to areas with more limited laboratory capacity," he writes. "The centre is definitely innovating towards collaboration with key stakeholders on all sides of the equation besides diagnostic testing - research into more effective and efficient treatments, successful program planning, implementation and evaluation, overarching policy and coalition building, outreach, cultural sensitivity and behavioural sciences to answer some of the difficult questions facing global health efforts." Tiago Villanueva Gutierrez Arruda Marques, MD, Lisbon, Portugal

CMAJ 2013. DOI:10.1503/cmaj.109-4357 